Research Paper

\title{
The Effect of Aerobic Training With the Consumption of Probiotics on the Myelination of Nerve Fibers in Cuprizone-induced Demyelination Mouse Model of Multiple Sclerosis
}

Donya Sajedi $^{1}$ (D), Ramin Shabani ${ }^{1^{*}}$ (D), Alireza Elmieh ${ }^{1}$ (D)

1. Department of Physical Education and Sports Sciences, Faculty of Humanities, Rasht Branch, Islamic Azad University, Rasht, Iran

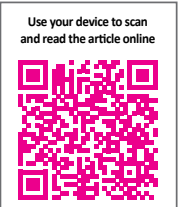

ctitation Sajedi, D., Shabani, R., \& Elmieh A. The Effect of Aerobic Training With the Consumption of Probiotics on the Myelination of Nerve Fibers in Cuprizone-induced Demyelination Mouse Model of Multiple Sclerosis. (2023). Basic and Clinical Neuroscience, 14(1), 73-86. http://dx.doi.org/10.32598/bcn.2022.3104.1

doi'http://dx.doi.org/10.32598/ben.2022.3104.1

Article info:

Received: 05 Jun 2021

First Revision: 10 Nov 2020

Accepted: 02 Jan 2022

Available Online: 01 Jan 2023

Keywords:

Myelination, Multiple sclerosis,

Exercise, Lactobacillus

plantarum, Cyclohexanes

\section{ABS TRACT}

Introduction: Extensive human and animal research shows that exercise has beneficial effects on multiple clinical outcomes for patients suffering from multiple sclerosis (MS). This research was conducted to examine the effect of aerobic exercise with probiotic consumption on the myelination of nerve fibers in a cuprizone-induced demyelination mouse model of MS.

Methods: Rats exposed to cuprizone (CPZ) for 13 weeks were subjected to motor and balance tests in week 5 . They ( 5 people in each group) were assigned to five groups of control (C), MS, MS with exercise (MS+Exe), MS with probiotic (MS+Pro), and MS with probiotic and exercise (MS+Pro+Exe) randomly. The exercise groups conducted aerobic exercises 5 days a week for 60 days. The rats received probiotics by gavage. Performance and balance tests were repeated when the eight-week protocol of exercise and probiotic consumption was finished. One day after these interventions, they were sacrificed to undergo biochemical and molecular biology assays.

Results: The results showed that Myelin basic protein (MBP) was increased in the MS+Pro+Exe, MS+Pro, and MS+Exe compared to the MS group $(\mathrm{P}<0.05)$.

The nestin mRNA showed an increase in MS+Pro+Exe, MS+Exe, and MS+Pro groups compared to the MS group, but this increase was not significant in MS+Pro+Exe and MS+Exe groups compared to the control and MS groups $(\mathrm{P}>0.05)$.

Conclusion: According to the results, lifestyle interventions can effectively alleviate demyelinating-inflammatory processes that happen in the brains of MS patients. 


\section{Highlights}

- Exercise can improve demyelination in mice.

- Consumption of probiotics can be effective in improving multiple sclerosis disease.

- Exercise can modify the gut microbiota.

\section{Plain Language Summary}

Multiple sclerosis (MS) is a common inflammatory disorder of the central nervous system (CNS) categorized by myelin loss and decadence of neurons in the brain and spinal cord. Cuprizone feeding causes reversible demyelination, predominantly of the corpus callosum in C57/B16 rats. The results of previous studies showed that exercise training and consumption of probiotics alone effectively reduce demyelination of the nerve fibers. In this study, considering that the elimination of cuprizone from rats' food causes remyelination, its application continued until the end of the protocol.In this study, we emphasize exercise training and the consumption of probiotics. Our results showed that in addition to the effect of exercise training and consumption of probiotics on the remyelination of nerve fibers, both of these interventions have a greater effect on it.

\section{Introduction}

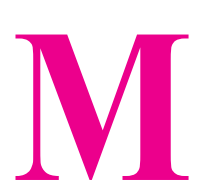

ultiple sclerosis (MS) is the most common demyelinating disease of the Central Nervous System (CNS) causing neurological disability, mostly in young adults (Eckstein et al., 2012). Women suffer from MS 2-3 times more often than men (Leray et al., 2016). MS patients show various physiological and psychological symptoms, e.g. cognitive and emotional problems, visual impairments, fatigue, general muscle weakness, and motor disturbances (Huang et al., 2017). Myelin sheath is a lipid-protein substance, which wraps nerve fibers (axons). The myelin cells, or the so-called oligodendrocytes, are the main ground for disease initiation. The term MS refers to the formation of scar tissues or plaques or lesions in certain parts of the brain, especially in the white matter (Rahmanzadeh et al., 2018). Infiltrating T cells and monocytes/macrophages are not only responsible for the death of the oligodendrocyte cells, but also employ brain resident immune cells, i.e. astroglia and microglia, which, in turn, both amplify and resolve inflammation (Popescu \& Lucchinetti, 2012).

Inflammatory infiltration into the CNS destroys major myelin proteins, such as Myelin basic protein (MBP) (Popescu \& Lucchinetti, 2012). This inflammatory process causes demyelination, axonal damage, and subsequently progressive hind-limb paralysis (Robinson et al., 2014).
In MS, where lesions throughout the CNS are demyelinated by the damage to oligodendrocytes and their myelin sheaths, spontaneous myelin repair was identified years ago. More recent studies have revealed that remyelination happens earlier and more extensively than previous studies have reported (Brück et al., 2003). The expression of the intermediate filament protein nestin has been utilized as a marker for neural stem and progenitor cells in the ventricular and subventricular zones (Hockfield \& McKay, 1985). Radial glia (Hockfield \& McKay, 1985), which are substrates for migration and can give rise to neurons (Noctor et al., 2001), also express nestin. The second intronic enhancer of nestin signifies the expression of the gene to neural tissues (Zimmerman et al., 1994).

Most clinical studies have reported the benefits of exercise training for molecular, histopathological, and behavioral abnormalities in MS patients and animal models of MS (Motl \& Pilutti, 2012). The anti-inflammatory effects of exercise training are also documented in MS patients (Florindo, 2014). Exercise-based protocols for animal models are composed of voluntary wheel running, forced treadmill running, or swimming. Although both voluntary and forced exercise paradigms are effective preconditioning tools for the CNS (Zhang et al., 2011), forced exercise has been found to outperform voluntary exercise in promoting neuroprotection by altering brain metabolism (Kinni et al., 2011). Moreover, forced exercise protocols allow modifying the intensity, duration, and frequency of the program and may motivate a human's schedule of gym exercises (Zhang et al., 2011). 
Also, it is suggested that improving adaptation to stress responses and promoting neuroprotection requires exercise periods of at least three weeks (Adlard et al., 2005).

The gut microbiome is claimed to cause several autoimmune disorders, including inflammatory bowel disease, rheumatoid arthritis, and MS (Bhargava \& Mowry, 2014). Recent research has revealed a correlation between intestinal T helper 17 (Th17) cell abundance with changes in gut microbiota composition and increased disease activity in MS (Cosorich et al., 2017). Thus, it can be said that MS patients can potentially benefit from, for instance, manipulating the gut microbiome with probiotics. Probiotics are live and non-pathogenic microorganisms found in certain foods. Some of these microorganisms belong to selected bacterial strains, $\mathrm{Lac}$ tobacillus (Goudarzvand, 2016).

Some evidence suggests that exercise may modify the microbiota (Queipo-Ortuño et al., 2013). Accordingly, a study on elite rugby players reported that exercise increased gut microbiota richness and diversity (Clarke et al., 2014). Also, recent studies on animals have shown that controlled training also has some beneficial effects on the gut microbiome of obese and hypertensive rats (Petriz et al., 2014) and in obese rats with high-fat dietinduced phenotype (Kang et al., 2014).

The cuprizone (CPZ) model provides a reproducible method to investigate the processes of primary demyelination, inflammation, axonal damage, and myelin repair/ remyelination when no peripheral immune system activation is observed. As a mitochondrial copper-chelating agent, CPZ selectively targets mature oligodendrocytes of the CNS, especially those of the corpus callosum. Demyelination completes after five weeks of CPZ intoxication, accompanied by massive microgliosis, astrocytosis, and axonal damage (Hibbits et al., 2009). Furthermore, removing CPZ from animals' diets improves remyelination (Heckers, 2018).

The present study is an attempt to explore the impact of exercise with Lactobacillus plantarum (L. plantarum) probiotics on motor deficits associated with the CPZ model and myelination. To deal with this issue, the research adopted a model of exercise training that can mimic endurance training in humans and is associated with increased wellness in both healthy individuals and those with neurodegenerative disorders.

\section{Materials and Methods}

\section{Animals}

A total of 25 female C57BL/ 6 rats ( 8 weeks old, $20 \pm 4$ $\mathrm{g}$ ), procured from the Pasteur Institute of Iran, were transferred to the histogenotech lab of Tehran, Iran. They were kept in communal cages at $22^{\circ} \mathrm{C} \pm 1{ }^{\circ} \mathrm{C}$ under a 12 -h photoperiod (lights on at 07:00) with full-time access to food and water. All procedures were approved by the Islamic Azad University, Rasht Branch (Rasht City, Iran) and Institutional Animal Care and Use Committee (Ethical Code: IR.IAU.RASHT.REC.1399.028) and complied with the National Institute of Health $(\mathrm{NIH})$ guidelines. Behavioral experiments were conducted from 8 AM to 4 PM.

\section{Experimental design and animal group}

Five rats in each group were randomly divided into five groups, control (C) that received no intervention, MS exposed to CPZ, MS with exercise (MS+Exe), MS with probiotic (MS+Pro), and MS with probiotic and exercise (MS+Pro+Exe).

\section{Cuprizone (CPZ) model}

Rats were exposed to $\mathrm{CPZ}$ for 13 weeks. To induce demyelination, the rats were fed on a diet composed of $0.2 \% \mathrm{CPZ}$ (bis cyclohexylidenehydrazide; Sigma-Aldrich Inc., St. Louis, MO, USA) mixed with rodent food triturated pellets (Gudi et al., 2014b). Since remyelination is increased in the absence of CPZ in the diet of animals (Heckers, 2018), its use was continued until the end of the protocol. Therefore, the daily food of the animals contained during the exercise period.

\section{Beam test}

The mice were subjected to the mobility and balance tests by the beam test in week 5 of CPZ feeding and at the end of the eight-week protocol of exercise and probiotic consumption. They were trained to go from the suspended end of a narrow beam (120 cm length, $7 \mathrm{~cm}$ width, elevated $100 \mathrm{~cm}$ above a thick foam cushion) into a goal box $(24.5 \times 20 \times 18)$. Two observers recorded the number of foot slips to start a movement. A foot slip is defined as one single paw going down by $1.5 \mathrm{~cm}$ or more beneath the beam surface (Mu et al., 2011). 


\section{Probiotic supplement}

L. plantarum strain Persian type culture collection (PTCC) 1058 was chosen by the Iranian Research Organization for Science and Technology, Tehran City, Iran for testing. After the bacteria were cultured and their purity was confirmed, they were anaerobically propagated in de Man, Rogosa, and Sharpe (MRS) broth at $37^{\circ} \mathrm{C}$ for $48 \mathrm{~h}$ at a volume of $500 \mathrm{~mL}$. After $48 \mathrm{~h}$, they were centrifuged, washed with phosphate-buffered saline (PBS), and re-centrifuged to be isolated from the growth media. The treatments were made for each animal with $1.5 \mathrm{~mL}$ of resuspended bacteria in saline at a concentration of $10^{8}$ colony-forming units (CFU) $/ \mathrm{kg}$. The probiotic was gavaged 5 times a week $1 \mathrm{~h}$ after exercise training.

\section{Treadmill exercise training}

The mice were subject to physical training and performance tests on an 8-lane treadmill (Tajhiz Gostar Omid Iranian, Iran). Before the exercise performance tests and the training program were initiated, the mice were gotten acquainted with treadmill running for 10 minutes on three consecutive days $(8 \mathrm{~cm} / \mathrm{s})$. The performance was tested before training and at the end of the 8-week training protocol. A 72-h interval was considered between these two performance tests.

\section{Exhaustion speed performance test}

The exhaustion speed performance test was employed to measure the maximal running speed. For this test, the rats ran for $8 \mathrm{~cm} / \mathrm{s}$ and then increased the speed to $2 \mathrm{~cm} / \mathrm{s}$ per minute until exhaustion [modified from (Qi et al., 2011)]. Exhaustion was recorded when the mice could not or declined to keep on even if encouraged with a bottle brush or a small puff of air.

\section{Exercise tolerance performance test}

To evaluate the exercise tolerance, the exercise tolerance performance test was employed for which each rate was individually run at a speed of $30 \mathrm{~cm} / \mathrm{s}$ on a rodentspecific treadmill [modified from (Ritchie et al., 2014)]. Exhaustion was defined as above.

\section{Exercise training protocol}

The rats were exposed to an 8-week treadmill running, 5 days per week, 1 session per day at a rate of $23 \mathrm{~cm} / \mathrm{s}$. This training speed is equivalent to an exercise intensity of $55 \%-60 \%$ of maximal speed based on the baseline exercise speed performance tests. An incremental exercise training protocol was applied to the trained rats.
Each training session consisted of a 5-minute warm-up at $8 \mathrm{~cm} / \mathrm{s}$ followed by 10 minutes of training in week 1 and 20 minutes of training in week 2 . In the subsequent six weeks, the warm-up was followed by 30 minutes of training at $23 \mathrm{~s}$ per minute. The potential intervening factors, including differences in stress, sound, and light exposure, were minimized by leaving the sedentary control rats on the treadmill without running for the same duration as the exercise groups. All animals were included in the experiments (Figure 1).

The rats were initially exposed to CPZ for 5 weeks. The performance tests and beam tests were carried out before the training. Then, the eight-week training protocol began. The training protocol included 10 minutes of training for the first week, 20 minutes of training for the second week, and 30 minutes of training with an intensity of $23 \mathrm{~cm} / \mathrm{s}$ during the next 6 weeks. During the eightweek training period, the mice were given probiotics by gavage 5 days a week. One 1-h break existed between activity and probiotics. The performance tests and beam tests were replicated when the eighth week was over.

\section{Tissue preparation}

One day after the eight weeks of the exercise training protocol and the intake of probiotics, the rats were sacrificed under $\mathrm{CO}_{2}$ gas. The whole brain was exposed and divided anteriorly-posteriorly in bregma place using a coronal section. The anterior part was employed for western blot and real-time polymerase chain reaction (PCR) and the posterior part for histological assessment

Histological evaluation and luxol fast blue (LFB) staining

To assess myelination, the corpus callosum was eliminated after the rats were sacrificed and fixed overnight. Then, the samples were dehydrated in ascending alcohol series, rinsed with xylene, and infiltrated with paraffin. Afterward, all the paraffin-embedded specimens were coronally sectioned at a thickness of $5 \mu \mathrm{m}$. The samples were stained with luxol fast blue (LFB) (Sigma-Aldrich) according to the manufacturer's instructions. Briefly, a graded serial of ethanol to $1 \%$ LFB solution in $0.05 \%$ acetic acid and $96 \%$ ethanol was used to deparaffinized and transform corpus callosum sections. Sections were put in LFB solution at $56^{\circ} \mathrm{C}$ overnight, then rinsed in $95 \%$ ethyl alcohol and distilled water before differentiating in $70 \%$ ethyl alcohol. After differentiation in the lithium carbonate solution, they were placed in distilled water again and counterstained. The samples were analyzed under a light microscope (Labomed, USA). Image 


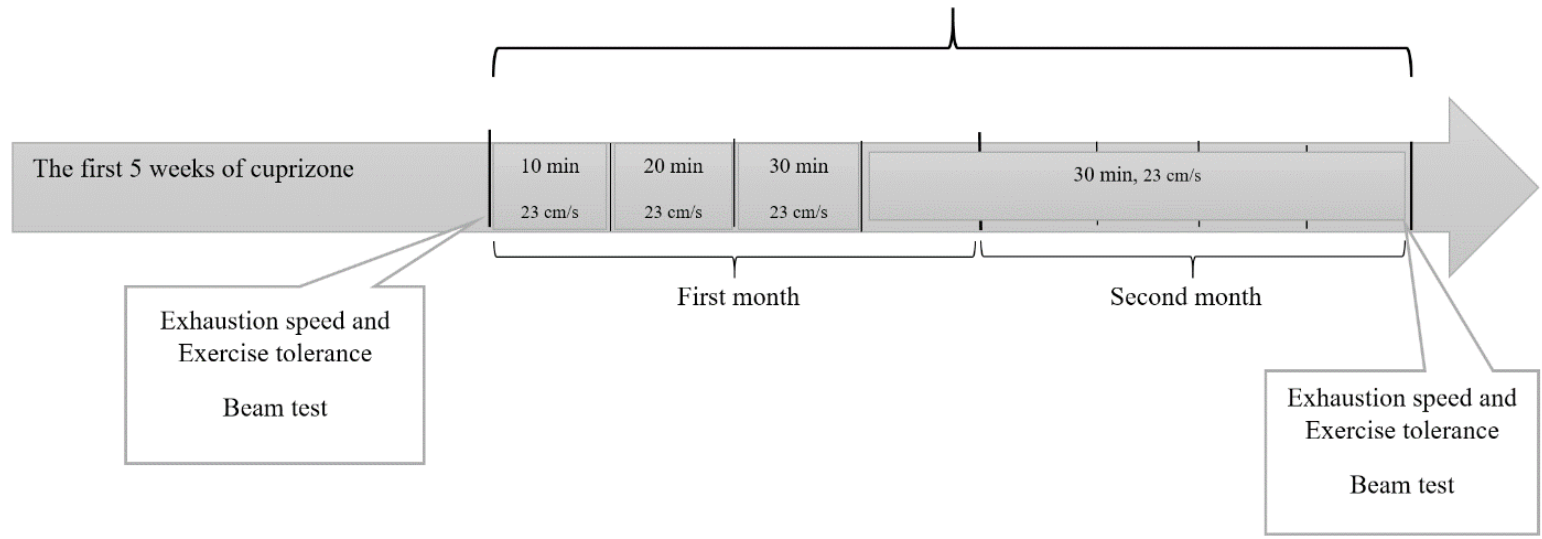

Figure 1. Experimental protocol for exploring the impacts of exercise training

NEUR SCIENCE

J software was used to evaluate of amount of myelin. For this reason, the amount of dark blue to whole tissue in each section was assessed.

Striatal total protein extracts preparation and western blot (WB)

The protein concentration of each sample was measured with Lowry assay to ensure that equal amounts were loaded during western blotting. First, in the stage of running the gel, mix the protein sample with the sample buffer and boil for 5-10 minutes, and then run on polyacrylamide gel for 2-3 h at 100 volts. After running, the gel was prepared for the transfer stage. In the transfer step, we first cut the nitrocellulose paper as needed and put it inside the transfer buffer along with the device pads and sponges. To cut the nitrocellulose paper, we used a clean scalpel and avoided touching the paper with our hands. Then, the nitrocellulose paper was inserted with gel and sponges and the device pads inside a tank and exposed to 350 amps electrophoresis for $1 \mathrm{~h}$. After transfer, the nitrocellulose paper was washed with a tris-buffered saline (TBS) buffer for 5-10 minutes. At this stage, the nitrocellulose paper was immersed in the TBS buffer (or blocking) for $1 \mathrm{~h}$ at room temperature, and gently shaken. Then, the nitrocellulose paper was washed several times using a TBS buffer. Then, the nitrocellulose paper was incubated for 1-2 $\mathrm{h}$ at room temperature with the primary antibody diluted in the TBS buffer (antibody accuracy was 1000/1). The nitrocellulose paper was rinsed several times using a TBS buffer. Then, it was incubated for 1-2 $\mathrm{h}$ at room temperature with a secondary antibody diluted in the TBS buffer (antibody accuracy was 3000/1). The nitrocellulose paper was rinsed several times using a TBS buffer. This step of nitrocellulose paper is developed on the photographic film using the emergence and fixation solution using ECL in a dark room. Finally, after the band appeared, the paper can be washed with distilled water.

Messenger ribonucleic acid (MRNA) gene expression analysis

The corpus callosum tissue, prepared by Qiazol (Qiazol lysis reagent, USA), was prepared in completely sterile conditions on the ice. The concentration and purity of RNA were determined by the ratio of the absorbance at $260 \mathrm{~nm}$ over that at $280 \mathrm{~nm}$ (A 260/A 280) using a nanodrop ND-100 spectrophotometer (Thermo Scientific, Waltham, MA, USA). The RevertAid cDNA synthesis kit (Thermo Scientific, USA) was used at a volume of 10 $\mu \mathrm{L}$ to convert RNA into complementary DNA (cDNA) based on the manufacturer's recommendations. The extracted RNA samples, previously infected with genomic DNA, were treated using deoxyribonuclease I (DNase I). Polymerase chain reaction (PCR) was amplified by $2 \mu \mathrm{L}$ of the cDNA synthesis reaction, $12.5 \mu \mathrm{L}$ of RealQ Plus master mix green high ROX ${ }^{\mathrm{TM}}$ (Amplicon, Denmark), $0.2 \mu \mathrm{L}$ of each forward and reverse primers (at the concentration of 10 pico-molar), and $10.1 \mu \mathrm{L}$ of distilled water. The primers were prepared by the Primer 3 software package and verified by the NCBI BLAST Tool. Table 1 presents the primers used here.

Thermocycling conditions were $95^{\circ} \mathrm{C}$ for 15 minutes, followed by 40 cycles of denaturation at $95^{\circ} \mathrm{C}$ for $15 \mathrm{~s}$ and $60^{\circ} \mathrm{C}$ for 1 minute. The $\Delta \mathrm{CT}$ method (Equation 1 which is based on the yield and difference in CT, was employed to specify the relative expressions for the nestin gene.

\section{1. $\Delta \mathrm{CT}=\mathrm{CT}$ target $-\mathrm{CT}$ reference}

CT: Threshold cycle 
$\triangle \mathrm{CT}$ and CT: In fact, to obtain the ratio of the desired gene to the reference gene (to normalize the data). The CT samples in the target gene were compared to the internal control of the CT gene. The real-time PCR was conducted on the ABI Stepone (applied biosystems, USA) detection system. Electrophoresis and melting curve analysis was employed to re-examine the specificity of the real-time PCR reaction.

\section{Statistical analysis}

GraphPad PRISM software, version 5 was used for statistical analysis. The differences in means among groups for all variables were compared using a one-way analysis of variance (ANOVA). The between-group differences of means were distinguished by Tukey's multiple comparison tests. All values are expressed as Mean \pm SD. The statistical significance level was set at $\mathrm{P}<0.05$ for all tests.

\section{Results}

The effect of aerobic training with the consumption of probiotics in the Beam test

The results showed that the error level in the beam test was significantly decreased in the MS+Pro+Exe and $\mathrm{MS}+$ Exe compared to the MS group $(\mathrm{P}<0.05)$. Also, the error level in MS+Pro group decreased compared to the MS group but the difference was not significant $(\mathrm{P}>0.05)$ (Figure 2).

The effect of aerobic training with the consumption of probiotics in the performance tests

To examine the efficacy of the training protocol, exhaustion speed, and exercise tolerance performance tests were performed. The results of both tests in the MS+Pro+Exe, $\mathrm{MS}+\mathrm{Exe}$, and MS+Pro groups showed a significant increase in the exhaustion speed, and exercise tolerance compared to the MS group $(\mathrm{P}<0.05)$ (Figure 3, 4).

Induction of demyelination in cuprizone (CPZ)fed rats

The histological changes in this study were performed using LFB staining method. Figure 5, 6 shows the myelination changes in different groups. As shown in the Figure, the myelination of the nerve fibers was significantly $(\mathrm{P}<0.05)$ higher in the MS+Pro+Exe, MS+Pro, and MS+Exe groups than in the MS group.

\section{Western blot analysis}

The results showed that MBP expression was significantly increased in the MS+Pro+Exe, MS+Pro, and MS+Exe compared to the MS group ( $\mathrm{P}<0.05)$. However, a combination of probiotics with exercise training protocols showed the highest increase in MBP (Figure 7).

\section{Beam test}

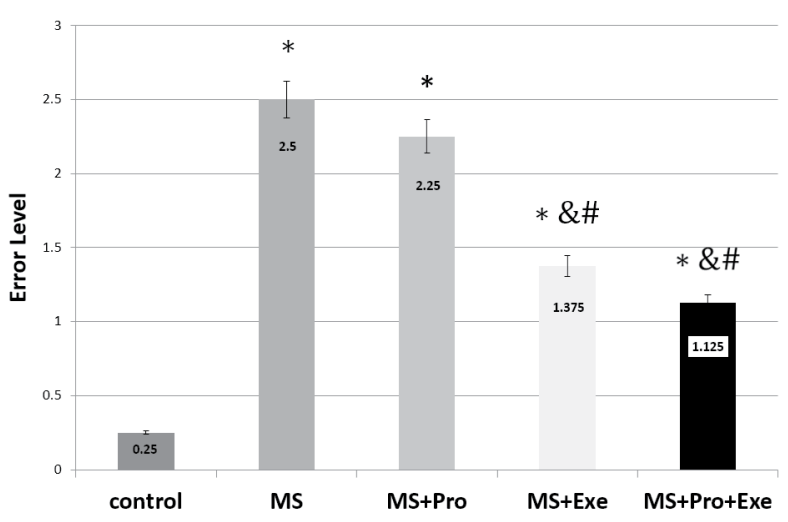

Figure 2. The Beam test results in different study groups

The values are displayed as the Means \pm SD. The difference signs represent statistically significant differences between the mean values $(\mathrm{P}<0.05)$ and the same signs are not significant.

One-way ANOVA analysis and Tukey post-hoc in all groups were significantly different from the control group ( $\mathrm{P}<0.05$ vs. control group), and MS+Exe and MS+Pro+Exe groups were significantly different from the MS and MS+Pro groups (P<0.05 vs. MS group and ${ }^{\#} \mathrm{P}<0.05$ vs. MS+Pro group).

MS+Pro: Multiple sclerosis plus probiotic; MS+Exe: Multiple sclerosis plus exercise; MS+Pro+Exe: Multiple sclerosis plus probiotic plus exercise. 


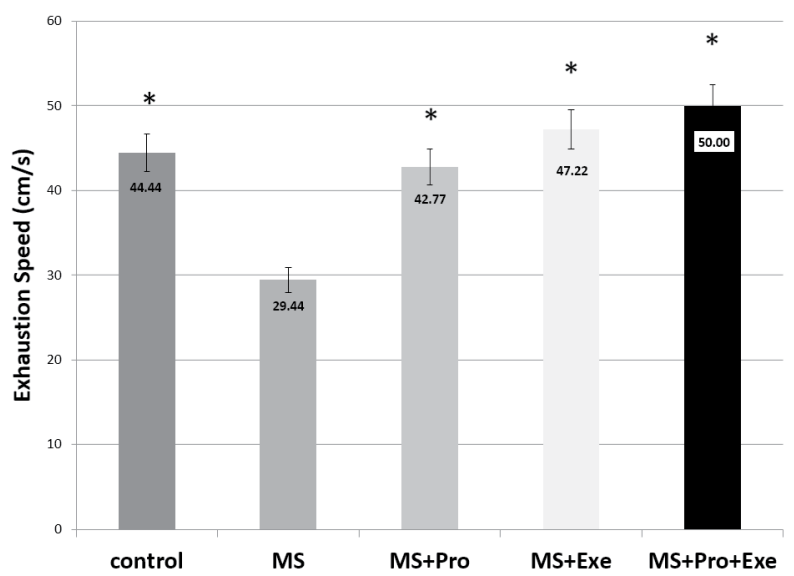

Figure 3. The exhaustion speed performance test results in different study groups

The values are shown as the Mean $\pm \mathrm{SD}$.

One-way ANOVA analysis and Tukey post-hoc in all groups were significantly different from the MS group ( ${ }^{*} \mathrm{P}<0.05$ vs. MS group).

MS+Pro: Multiple sclerosis plus probiotic; MS+Exe: Multiple sclerosis plus exercise; MS+Pro+Exe: Multiple sclerosis plus probiotic plus exercise.

\section{Expression of nestin}

The results showed that the gene expression of nestin was higher in MS+Pro+Exe, MS+Exe, and MS+Pro groups than in the MS group, but the difference was not significant $(\mathrm{P}>0.05)$ (Figure 8$)$.

\section{Discussion}

In this study, the effectiveness of aerobic exercise with the probiotic consumption on myelination, histological changes, and the expression of the gene nestin in the corpus callosum for the animal model of demyelination using CPZ administration was investigated. The present

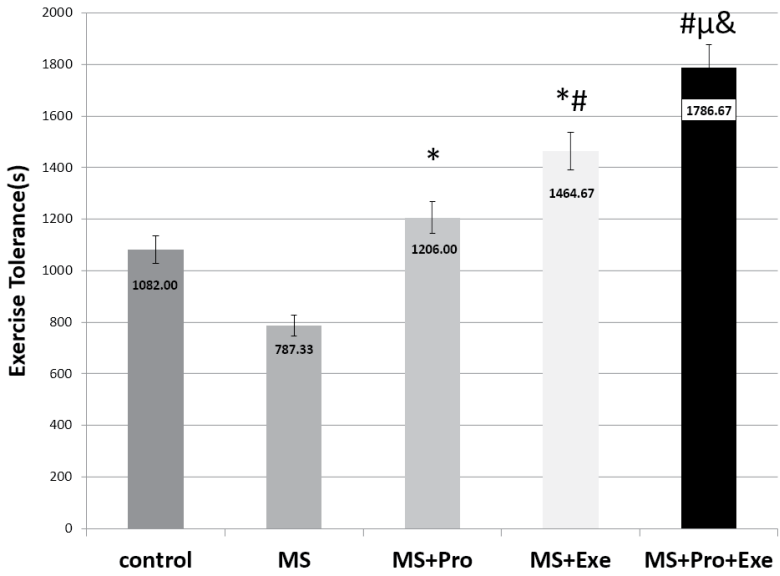

Figure 4. The exercise tolerance performance test resulting in different study groups

NEUR SCIENCE

The values are displayed as the Means \pm SD. The difference signs represent statistically significant differences between the mean values $(\mathrm{P}<0.05)$ and the same signs are not significant.

One-way ANOVA analysis and Tukey post-hoc in MS+Pro and MS+Exe groups were significantly different from the MS group $\left({ }^{*} \mathrm{P}<0.05\right.$ vs. MS group), MS+Exe and MS+Pro+Exe groups were significantly different from the control group ( ${ }^{*} \mathrm{P}<0.05$ vs. control group), MS+Pro+Exe group was significantly different from the MS group ( $\mu \mathrm{P}<0.05$ vs. MS group), and MS+Pro+Exe group was significantly different from the MS+Pro group ( $\mathrm{P}<0.05$ vs. MS+Pro group).

MS+Pro: Multiple sclerosis plus probiotic; MS+Exe: Multiple sclerosis plus exercise; MS+Pro+Exe: Multiple sclerosis plus probiotic plus exercise. 

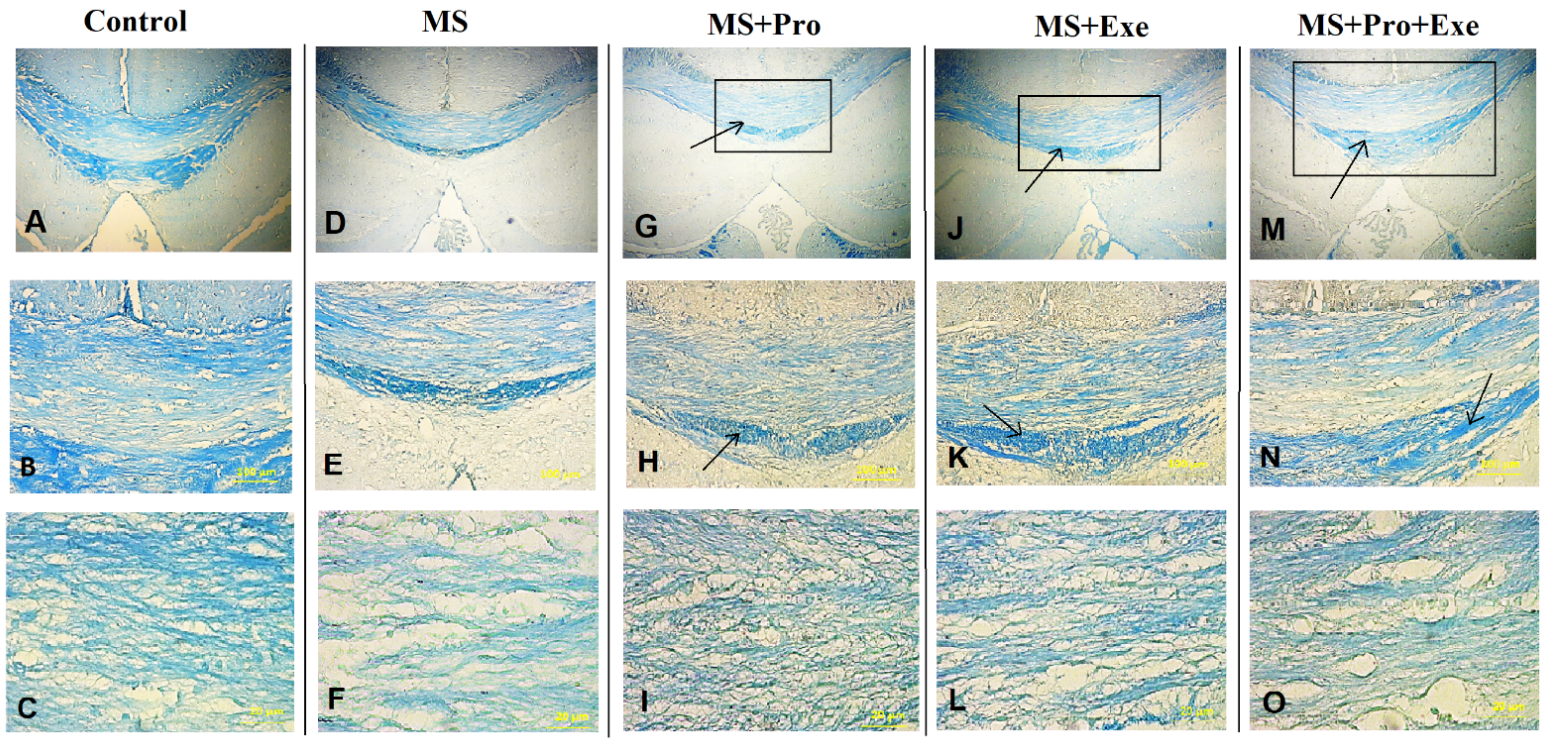

Figure 5. Histological changes in myelination in different treatment groups

NEUR SCIENCE

The above images show the longitudinal cross-section of nerve fibers stained with LFB staining. Arrows indicate areas of myelination. Bright areas indicate a decrease in myelin density in the MS group (D, E, and F) and the myelination of the nerve fibers is lower in this group than in the other groups. In the intervention groups (MS+Exe, MS+Pro, and MS+Pro+Exe), an increased amount of myelin is observed in the images, respectively. The MS+Pro+Exe group (M, N, and O), has the highest rate of myelination compared to the control group. Also in this group, the myelination of the nerve fibers is arranged in a regular, coherent structure. The diameter of the nerve fibers is also higher in this group than in the other groups.

MS+Pro+Exe: Multiple sclerosis plus probiotic plus exercise; MS+Pro: Multiple sclerosis plus probiotic; MS+Exe: Multiple sclerosis plus exercise.

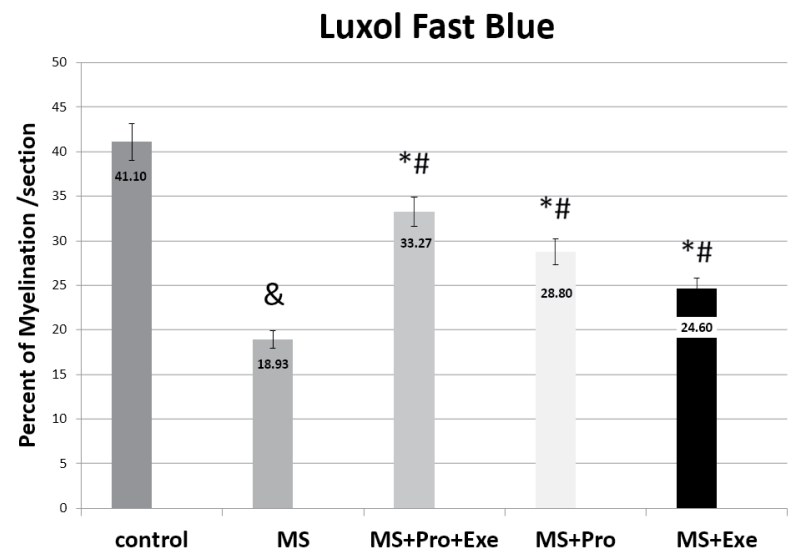

NEUR SCIENCE

Figure 6. The myelination of the nerve fibers in different study groups, observed percentages of myelination, blue fibers in different groups

The values are displayed as the Means \pm SD. The difference signs represent statistically significant differences between the mean values $(\mathrm{P}<0.05)$ and the same signs are not significant.

One-way ANOVA analysis and Tukey post-hoc in MS+Pro+Exe, MS+Pro and MS+Exe groups were significantly different from the control and MS groups ( ${ }^{*}<<0.05$ vs. control group and ${ }^{\#} \mathrm{P}<0.05$ vs. MS group), and MS group was significantly different from the control group ( $\mathrm{P}<0.05$ vs. control group).

MS+Pro+Exe: Multiple sclerosis plus probiotic plus exercise; MS+Pro: Multiple sclerosis plus probiotic; MS+Exe: Multiple sclerosis plus exercise. 
Table 1. The characteristics of the primers used in the present study

\begin{tabular}{cc}
\hline Gene & Sequence \\
\hline NES & F: 5' CCCCTTTCTTCTGTGTCTCACC $3^{\prime}$ \\
& R: 5' TCACTCATCATTGCTGCTCCTCT 3' \\
B-Actin & F: 5' TCAGAGCAAGAGAGGCATCC 3' \\
& R: 5' GGTCATCTTCTCACGGTTGG 3' \\
\hline
\end{tabular}

NES: Nestin

NEUR:SCIENCE

study used the CPZ model to induce demyelination. It is a famous experimental animal model used in research on the pathophysiological mechanisms of MS (Kipp et al., 2017). The results showed that aerobic exercise significantly improved motor deficits in animal models of MS. The MS+Exe group showed more improvement in motor function than the MS+Pro group. However, the highest rate of motor improvement was observed in the MS+Pro+Exe group. This is consistent with a previous study (Mandolesi et al., 2019) that has shown that exercise can improve clinical scores in rats with demyelination.

The study revealed an increase in the amount of MBP and myelination with exercise and probiotic consumption. Also, the myelination rate and MBP level in MS+Pro group increased more than in the MS +Exe group. A study reported the significant effect of probiotic treatments on clinical improvement, which was ascribed to the retention of myelin content and the reduction of astrocytosis and $\mathrm{CD}^{+} \mathrm{T}$ cell infiltration (Consonni et al., 2018).

Physical exercise has been reported to influence myelination variously depending on the analyzed brain area (Tomlinson et al., 2018). Functional recovery, enhanced by exercise, was associated with limited myelin destruction and the loss of myelin-associated proteins in white matter tracts of the corpus callosum and reduced axonal pathology. Moreover, exercise significantly intensified innate immune response, i.e. microgliosis and, to a lesser extent, astrogliosis. In rodents, exercise is related to significant changes in brain anatomy, including its weight and size, hippocampal neurogenesis and synaptogenesis (Van Praag et al., 2000), and synaptic plasticity (Patten et al., 2013). Myelination and remyelination use various
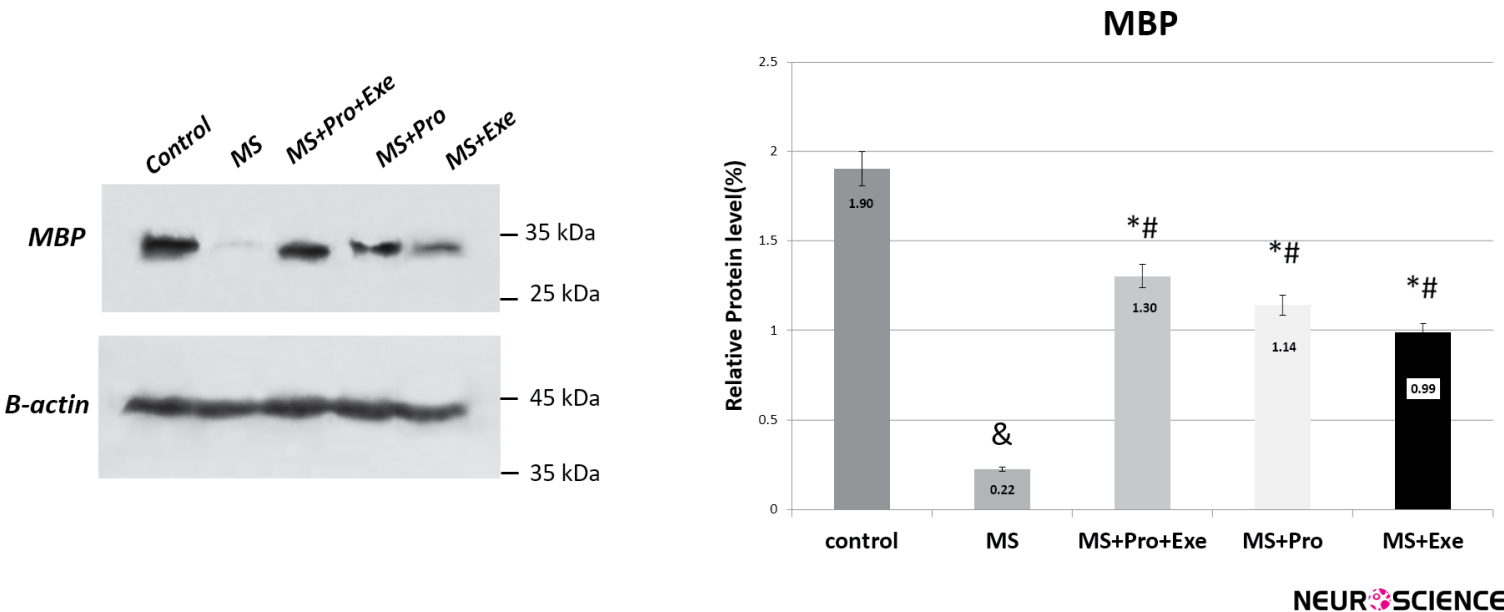

Figure 7. The expression of myelin basic protein $(\mathrm{mbp})$ in the corpus callosum area of different study groups

$\beta$-Actin was used as a loading control. The values are displayed as the Means \pm SD. The difference signs represent statistically significant differences between the mean values $(\mathrm{P}<0.05)$ and the same signs are not significant.

One-way ANOVA analysis and Tukey post-hoc in MS+Pro+Exe, MS+Pro, and MS+Exe groups were significantly different from the control and MS groups ( ${ }^{*} \mathrm{P}<0.05$ vs. control group and ${ }^{\#} \mathrm{P}<0.05$ vs. MS group), and MS group was significantly different from the control group ( $\& \mathrm{P}<0.05$ vs. control group).

MS+Pro+Exe, multiple sclerosis plus probiotic plus exercise; MS+Pro, multiple sclerosis plus probiotic; MS+Exe, multiple sclerosis plus exercise. 


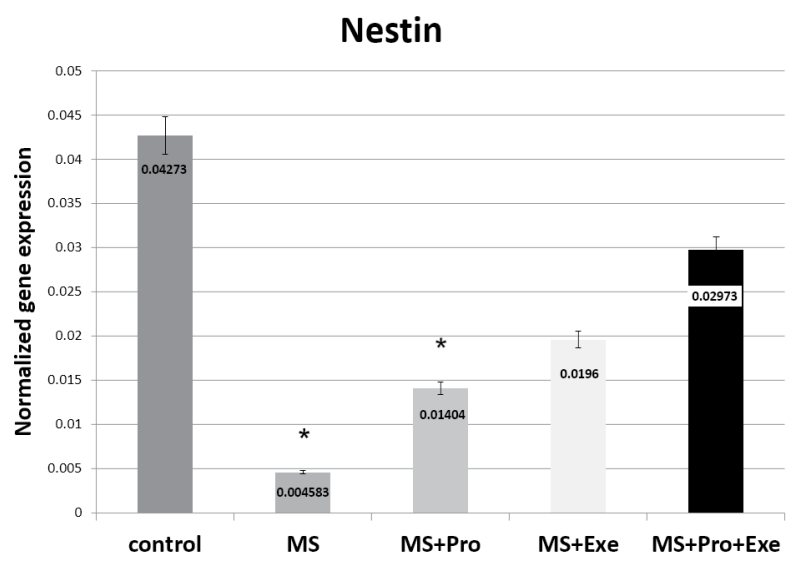

NEUR:SCIENCE

Figure 8. The messenger ribonucleic acid (mRNA) expression of nestin in the corpus callosum area of different study groups

The values are shown as the Means $\pm \mathrm{SD}$.

One-way ANOVA analysis and Tukey post-hoc in MS and MS+Pro groups were significantly different from the control group $\left({ }^{*} \mathrm{P}<0.05\right.$ vs. control group)

MS+Pro, multiple sclerosis plus probiotic; MS+Exe, multiple sclerosis plus exercise; MS+Pro+Exe, multiple sclerosis plus probiotic plus exercise.

complex mechanisms to differentiate oligodendrocyte precursor cells through different stages into mature myelinating oligodendrocytes (Bercury \& Macklin, 2015). In the CNS, the activation of microglia and astrocytosis are crucial components of the lesion environment that can influence the demyelination process, and these cells have been crucially involved in demyelination/remyelination processes during CPZ (Gudi et al., 2014a). Nonetheless, long-term exercise-based protocols improve immune function. As far as macrophages are concern, these effects can be related to the higher phagocytic activity (Sugiura et al., 2001) or accelerated phenotype switching leading to quicker wound healing (Goh \& Ladiges, 2014). Although isolated inflammation is not effective in changing the number of oligodendrocyte precursor cells, it enlarges the cell bodies and increases the number of the processes (Di Bello et al., 1999) and young macrophages recruited during remyelination, which facilitates the differential of oligodendrocyte precursor cells by removing inhibitory myelin debris (Ruckh et al., 2012). Accordingly, the modulation of immune functions plays a key role in myelin regenerative processes contributing to the remyelination course. These data suggest that some oligodendrocyte precursor cells die between peak and chronic disease and do not remyelinate axons. Furthermore, proliferation, migration, differentiation, and remyelination are the behaviors of oligodendrocyte precursor cells that can be modulated not just by the CNS disease conditions and trauma lesions but also by environmental changes and physical activity training. They may even sometimes act via opposite directing forces (Ehninger et al., 2011). In fact, in demyelinating lesion models in which remyelination was observed, a relationship was detected between the decreased number of oligodendrocyte precursor cells and the myelin repair (Watanabe et al., 2002).

The study revealed an increase in nestin levels after aerobic training and probiotic consumption. The amount of nestin in the MS+Exe group increased more than in the MS+Pro group. Various types of CNS damage are followed by so-called reactive neurogenesis, i.e. the formation of new neurons to replace the ones that have been lost by the damaging factor (Parent, 2003). Nestin expression has been observed to increase in some pathological states, such as inflammation (Cameron \& Mckay, 2001), and others. Nestin expression in astrocytes is detected one day after damage and continues for one month. Astrocytes may play a role in neuroprotection by forming a glial barrier/scar around the damage zone (Nakamura et al., 2003).

Cholinergic neurons, especially those with nestin as a cofactor, do not immediately die after a neurotoxic event, but rather they enter an atrophic quiescent state in which they do not express the enzymes required to maintain cholinergic transmission. On the contrary, if cholinergic neurons are exposed to nerve growth factor (NGF) (Nagahara et al., 2009) or brain-derived neurotrophic factor (BDNF) (Morse et al., 1993) in a timely and repeated manner, a significant part of them (30-40\%) can be rescued from a pathological state. Therefore, nestin contributes to the dynamic remodeling of cells during development (Ruan, 
2001). Evidence shows that nestin has a cytoprotective function in the adult nervous system (Su et al., 2013). Exercise enhances neurotrophin levels, an enhancement that can last for weeks (Berchtold et al., 2010).

The present research revealed the effects of oral administration with L. plantarum. Since research on MS has mostly focused on the differences in the gut microbiome of MS patients compared to healthy people (Jhangi et al., 2014), some have dealt with changing the composition of gut microflora by probiotics that modulate the immune response in an animal model of MS (Ochoa-Reparaz et al., 2010). Nonetheless, growing evidence suggests that exercise can also alter gut microbial composition (Bermon et al., 2015). However, the efficacy of probiotic treatment on different diseases depends on bacterial and rats strains, host immunological state, experimental system, an adequate dose, and its mechanisms are not well-understood yet (Isolauri et al., 2001). In the present study, it was shown that L. plantarum can improve the myelination of the nerve fibers. The rate of improvement in myelination was higher in the MS+Pro+Exe group. We found that oral administration of these strains delayed the development of the disease and resulted in the suppression of MS progression. Probiotics inhibit the growth of other microorganisms or compete for receptors and binding sites with other intestinal microbes on the intestinal mucosa by producing antimicrobial agents or metabolic compounds (Collado et al., 2007; O'Shea et al., 2012). We know that the alteration in equilibrium between T helper 1 (Th1) and T helper 2 (Th2) responses are linked to the pathogenesis of a wide variety of autoimmune diseases, such as MS (Nosratabadi et al., 2016). Although it is argued that the Th17 cells have a key role to play in MS pathogenesis, the geography of their peripheral activation and expansion in humans is still unclear. In rats, effector Th17 cells are mostly activated in the small intestine (Ivanov et al., 2009), and the pathogenicity of myelin-reactive $T$ cells at the intestinal level and their capacity to trigger brain autoimmunity are intensified when they acquire a Th17 cell phenotype (Berer et al., 2011).

Many studies have documented that experimental autoimmune encephalomyelitis (EAE) is mediated by the Th1 and Th17 cells secreting pro-inflammatory cytokines (Kobayashi et al., 2010), while a relationship is observed between disease recovery and the increased levels of Th2 cytokines (Nosratabadi et al., 2016). An upregulation of these cytokines in probiotic-treated mice appears to stimulate a shift toward Th2 response which may be one of the mechanisms involved in the downregulation of the autoimmune response. Some previous studies have revealed that pro-inflammatory cytokines promote the recruitment of inflammatory cells (Haghmorad et al., 2017) while anti-inflammatory cytokines negatively regulate the secretion of pro-inflammatory cytokines (Moore et al., 2001). In this situation, damaged axons re-generate and the healing process of myelin and neurons is better organized (Sakalidou et al., 2011).

This study provides powerful evidence for a beneficial link between the animal and human systems as promising future therapeutics for MS.

\section{Conclusion}

In conclusion, our study demonstrates that aerobic exercise with probiotic consumption affects the main pathological symptoms in MS. These results suggest lifestyle interventions as a good non-pharmacological instrument to control disease progression against demyelinatinginflammatory processes in the brains of animals with MS. However, it is necessary to conduct further studies, especially on human subjects.

\section{Ethical Considerations}

\section{Compliance with ethical guidelines}

This study was approved by the Ethics Committee of the University of Islamic Azad University, Rasht Branch. (Code: IR.IAU.RASHT.REC.1399.028).

\section{Funding}

The paper was extracted from the $\mathrm{PhD}$ thesis of Donya Sajedi, Department of Physical Education and Sports Sciences, Faculty of Humanities, University of Islamic Azad University, Rasht Branch. (Registered Code: 117481815632161180099).

\section{Authors' contributions}

Conceptualization, methodology, software, investigation, resources, and data curation: Donya Sajedi and Ramin Shabani; Writing, original draft preparation, and funding acquisition: Donya Sajedi; Formal analysis, writing, review, and editing and project administration: Ramin Shabani; Visualization and supervision: Ramin Shabani and Alireza Elmieh.

\section{Conflict of interest}

The authors declared no conflict of interest. 


\section{References}

Adlard, P. A., Perreau, V. M., \& Cotman, C. W. (2005). The exercise-induced expression of BDNF within the hippocampus varies across life-span. Neurobiology of Aging, 26(4), 511-520. [DOI:10.1016/j.neurobiolaging.2004.05.006] [PMID]

Berchtold, N. C., Castello, N., \& Cotman, C. W. (2010). Exercise and time-dependent benefits to learning and memory. Neuroscience, 167(3), 588-597. [DOI:10.1016/j.neuroscience.2010.02.050] [PMID] [PMCID]

Bercury, K. K., \& Macklin, W. B. (2015). Dynamics and mechanisms of CNS myelination. Developmental Cell, 32(4), 447-458. [DOI:10.1016/j.devcel.2015.01.016] [PMID] [PMCID]

Berer, K., Mues, M., Koutrolos, M., Al Rasbi, Z., Boziki, M., \& Johner, C., et al. (2011). Commensal microbiota and myelin autoantigen cooperate to trigger autoimmune demyelination. Nature, 479(7374), 538-541. [DOI:10.1038/nature10554] [PMID]

Bermon, S., Petriz, B., Kajeniene, A., Prestes, J., Castell, L., \& Franco, O. L. (2015). The microbiota: An exercise immunology perspective. Exerc Immunol Rev, 21(21), 70-79. [Link]

Bhargava, P., \& Mowry, E. M. (2014). Gut microbiome and multiple sclerosis. Current neurology and neuroscience reports, 14(10), 492. [DOI:10.1007/s11910-014-0492-2] [PMID]

Brück, W., Kuhlmann, T., \& Stadelmann, C. (2003). Remyelination in multiple sclerosis. Journal of the Neurological Sciences, 206(2), 181-185. [DOI:10.1016/S0022-510X(02)00191-0] [PMID]

Cameron, H. A., \& Mckay, R. D. (2001). Adult neurogenesis produces a large pool of new granule cells in the dentate gyrus. Journal of Comparative Neurology, 435(4), 406-417. [DOI:10.1002/cne.1040] [PMID]

Clarke, S. F., Murphy, E. F., O'Sullivan, O., Lucey, A. J., Humphreys, M., \& Hogan, A., et al. (2014). Exercise and associated dietary extremes impact on gut microbial diversity. Gut Microbiota, 63(12), 1913-1920. [DOI:10.1136/gutjnl-2013-306541] [PMID]

Collado, M., Meriluoto, J., \& Salminen, S. (2007). Role of commercial probiotic strains against human pathogen adhesion to intestinal mucus. Letters in Applied Microbiology, 45(4), 454-460. [DOI:10.1111/j.1472-765X.2007.02212.x] [PMID]

Consonni, A., Cordiglieri, C., Rinaldi, E., Marolda, R., Ravanelli, I., \& Guidesi, E., et al. (2018). Administration of bifidobacterium and lactobacillus strains modulates experimental myasthenia gravis and experimental encephalomyelitis in Lewis rats. Oncotarget, 9(32), 22269-22287. [DOI:10.18632/oncotarget.25170] [PMID] [PMCID]

Cosorich, I., Dalla-Costa, G., Sorini, C., Ferrarese, R., Messina, M. J., \& Dolpady, J., et al. (2017). High frequency of intestinal TH17 cells correlates with microbiota alterations and disease activity in multiple sclerosis. Science Advances, 3(7), e1700492. [DOI:10.1126/sciadv.1700492] [PMID] [PMCID]

Di Bello, I. C., Dawson, M., Levine, J., \& Reynolds, R. (1999). Generation of oligodendroglial progenitors in acute inflammatory demyelinating lesions of the rat brain stem is associated with demyelination rather than inflammation. Journal of Neurocytology, 28(4-5), 365-381. [DOI:10.1023/ A:1007069815302] [PMID]
Eckstein, C., Saidha, S., \& Levy, M. (2012). A differential diagnosis of central nervous system demyelination: Beyond multiple sclerosis. Journal of Neurology, 259(5), 801-816. [DOI:10.1007/ s00415-011-6240-5] [PMID]

Ehninger, D., Wang, L. P., Klempin, F., Römer, B., Kettenmann, H., \& Kempermann, G. (2011). Enriched environment and physical activity reduce microglia and influence the fate of NG2 cells in the amygdala of adult mice. Cell and Tissue Research, 345(1), 69-86. [DOI:10.1007/s00441-011-1200-z] [PMID] [PMCID]

Florindo, M. (2014). Inflammatory cytokines and physical activity in multiple sclerosis. ISRN Neurology, 2014, 1-8. [DOI:10.1155/2014/151572] [PMID] [PMCID]

Goh, J., \& Ladiges, W. C. (2014). Exercise enhances wound healing and prevents cancer progression during aging by targeting macrophage polarity. Mechanisms of Ageing and Development, 139, 41-48. [DOI:10.1016/j.mad.2014.06.004] [PMID]

Goudarzvand, M., Khodaii, Z., \& Moghadam, S. S. (2016). Probiotics Lactobacillus plantarum and Bifidobacterium B94: cognitive function in demyelinated model. Medical Journal of The Islamic Republic of Iran, 30, 391. [PMCID]

Gudi, V., Gingele, S., Skripuletz, T., \& Stangel, M. (2014). Glial response during cuprizone-induced de- and remyelination in the CNS: Lessons learned. Frontiers in Cellular Neuroscience, 8, 73. [DOI:10.3389/fncel.2014.00073] [PMID] [PMCID]

Haghmorad, D., Mahmoudi, M. B., Salehipour, Z., Jalayer, Z., Brojeni, A. A. M., \& Rastin, M., et al. (2017). Hesperidin ameliorates immunological outcome and reduces neuroinflammation in the mouse model of multiple sclerosis. Journal of Neuroimmunology, 302, 23-33. [DOI:10.1016/j.jneuroim.2016.11.009] [PMID]

Heckers, S. (2018). [Astrocyte functions during cuprizone-induced de-and remyelination (German)] [PhD thesis]. Hannover: University of Veterinary Medicine Hannover. [Link]

Hibbits, N., Pannu, R., Wu, T. J., \& Armstrong, R. C. (2009). Cuprizone demyelination of the corpus callosum in mice correlates with altered social interaction and impaired bilateral sensorimotor coordination. ASN Neuro, 1(3), e00013. [DOI:10.1042/AN20090032] [PMID] [PMCID]

Hockfield, S., \& McKay, R. D. (1985). Identification of major cell classes in the developing mammalian nervous system. Journal of Neuroscience, 5(12), 3310-3328. [DOI:10.1523/JNEUROSCI.05-12-03310.1985] [PMID] [PMCID]

Huang, W. J., Chen, W. W., \& Zhang, X. (2017). Multiple sclerosis: Pathology, diagnosis and treatments. Experimental and Therapeutic Medicine, 13(6), 3163-3166. [DOI:10.3892/ etm.2017.4410] [PMID] [PMCID]

Isolauri, E., Sütas, Y., Kankaanpää, P., Arvilommi, H., \& Salminen, S. (2001). Probiotics: Effects on immunity. The American Journal of Clinical Nutrition, 73(2), S444-S450. [DOI:10.1093/ ajcn/73.2.444s] [PMID]

Ivanov, I. I., Atarashi, K., Manel, N., Brodie, E. L., Shima, T., \& Karaoz, U., et al. (2009). Induction of intestinal Th17 cells by segmented filamentous bacteria. Cell, 139(3), 485-498. [DOI:10.1016/j.cell.2009.09.033] [PMID] [PMCID] 
Jhangi, S., Gandhi, R., Glanz, B., Cook, S., Nejad, P., \& Ward, D., et al. (2014). Increased archaea species and changes with therapy in gut microbiome of multiple sclerosis subjects (S24.001). Neurology, 82(10 Supplement), S24.001. [Link]

Kang, S. S., Jeraldo, P. R., Kurti, A., Miller, M. E. B., Cook, M. D., \& Whitlock, K., et al. (2014). Diet and exercise orthogonally alter the gut microbiome and reveal independent associations with anxiety and cognition. Molecular Neurodegeneration, 9, 36. [DOI:10.1186/1750-1326-9-36] [PMID] [PMCID]

Kinni, H., Guo, M., Ding, J. Y., Konakondla, S., Dornbos III, D. \& Tran, R., et al. (2011). Cerebral metabolism after forced or voluntary physical exercise. Brain Research, 1388, 48-55. [DOI:10.1016/j.brainres.2011.02.076] [PMID]

Kipp, M., Nyamoya, S., Hochstrasser, T., \& Amor, S. (2017). Multiple sclerosis animal models: A clinical and histopathological perspective. Brain Pathology, 27(2), 123-137. [DOI:10.1111/ bpa.12454] [PMID] [PMCID]

Kobayashi, T., Kato, I., Nanno, M., Shida, K., Shibuya, K., Matsuoka, Y., et al (2010). Oral administration of probiotic bacteria, Lactobacillus casei and Bifidobacterium breve, does not exacerbate neurological symptoms in experimental autoimmune encelphalomyelitis. Immunopharmacology and Immunotoxicology, 32(1), 116-124. [DOI:10.3109/08923970903200716] [PMID]

Leray, E., Moreau, T., Fromont, A., \& Edan, G. (2016). Epidemiology of multiple sclerosis. Revue Neurologique, 172(1), 3-13. [DOI:10.1016/j.neurol.2015.10.006] [PMID]

Mandolesi, G., Bullitta, S., Fresegna, D., De Vito, F., Rizzo, F. R., \& Musella, A., et al. (2019). Voluntary running wheel attenuates motor deterioration and brain damage in cuprizoneinduced demyelination. Neurobiology of Disease, 129, 102-117. [DOI:10.1016/j.nbd.2019.05.010] [PMID]

Moore, K. W., de Waal Malefyt, R., Coffman, R. L., \& O'Garra, A (2001). Interleukin-10 and the interleukin-10 receptor. Annual Review of Immunology, 19, 683-765. [DOI:10.1146/annurev.immunol.19.1.683] [PMID]

Morse, J. K., Wiegand, S. J., Anderson, K., You, Y., Cai, N., \& Carnahan, J., et al. (1993). Brain-derived neurotrophic factor (BDNF) prevents the degeneration of medial septal cholinergic neurons following fimbria transection. The Journal of Neuroscience, 13(10), 4146-4156. [DOI:10.1523/JNEUROSCI.13-10-04146.1993] [PMID] [PMCID]

Motl, R. W., \& Pilutti, L. A. (2012). The benefits of exercise training in multiple sclerosis. Nature Reviews Neurology, 8(9), 487497. [DOI:10.1038/nrneurol.2012.136] [PMID]

Mu, S., OuYang, L., Liu, B., Zhu, Y., Li, K., \& Zhan, M., et al. (2011). Preferential interneuron survival in the transition zone of 3-NP-induced striatal injury in rats. Journal of Neuroscience Research, 89(5), 744-754. [DOI:10.1002/jnr.22591] [PMID]

Nagahara, A. H., Bernot, T., Moseanko, R., Brignolo, L., Blesch, A., \& Conner, J. M., et al. (2009). Long-term reversal of cholinergic neuronal decline in aged non-human primates by lentiviral NGF gene delivery. Experimental Neurology, 215(1), 153159. [DOI:10.1016/j.expneurol.2008.10.004] [PMID] [PMCID]

Nakamura, T., Xi, G., Hua, Y., Hoff, J. T., \& Keep, R. F. (2003). Nestin expression after experimental intracerebral hemorrhage. Brain Research, 981(1-2), 108-117. [DOI:10.1016/S00068993(03)02991-3]
Noctor, S. C., Flint, A. C., Weissman, T. A., Dammerman, R. S. \& Kriegstein, A. R. (2001). Neurons derived from radial glial cells establish radial units in neocortex. Nature, 409(6821), 714720. [DOI:10.1038/35055553] [PMID]

Nosratabadi, R., Rastin, M., Sankian, M., Haghmorad, D., \& Mahmoudi, M. (2016). Hyperforin-loaded gold nanoparticle alleviates experimental autoimmune encephalomyelitis by suppressing Th1 and Th17 cells and upregulating regulatory T cells. Nanomedicine: Nanotechnology, Biology and Medicine, 12(7), 1961-1971. [DOI:10.1016/j.nano.2016.04.001] [PMID]

Nosratabadi, R., Rastin, M., Sankian, M., Haghmorad, D., Tabasi, N., \& Zamani, S., et al. (2016). St. John's wort and its component hyperforin alleviate experimental autoimmune encephalomyelitis through expansion of regulatory T-cells. Journal of Immunotoxicology, 13(3), 364-374. [DOI:10.3109/154 7691X.2015.1101512] [PMID]

O'Shea, E. F., Cotter, P. D., Stanton, C., Ross, R. P., \& Hill, C. (2012). Production of bioactive substances by intestinal bacteria as a basis for explaining probiotic mechanisms: Bacteriocins and conjugated linoleic acid. International Journal of Food Microbiology, 152(3), 189-205. [DOI:10.1016/j.ijfoodmicro.2011.05.025] [PMID]

Ochoa-Reparaz, J., Mielcarz, D., Wang, Y., Begum-Haque, S., Dasgupta, S., \& Kasper, D., et al. (2010). A polysaccharide from the human commensal Bacteroides fragilis protects against CNS demyelinating disease. Mucosal Immunology 3(5), 487-495. [DOI:10.1038/mi.2010.29] [PMID]

Parent, J. M. (2003). Injury-induced neurogenesis in the adult mammalian brain. The Neuroscientist, 9(4), 261-272. [DOI:10.1177/1073858403252680] [PMID]

Patten, A. R., Sickmann, H., Hryciw, B. N., Kucharsky, T., Parton, R., \& Kernick, A., et al.. (2013). Long-term exercise is needed to enhance synaptic plasticity in the hippocampus. Learning $\mathcal{E}$ Memory, 20, 642-647. [DOI:10.1101/lm.030635.113] [PMID]

Petriz, B. A., Castro, A. P., Almeida, J. A., Gomes, C. P., Fernandes, G. R., \& Kruger, R. H., et al. (2014). Exercise induction of gut microbiota modifications in obese, non-obese and hypertensive rats. BMC Genomics, 15(1), 511. [DOI:10.1186/14712164-15-511] [PMID] [PMCID]

Popescu, B. F. G., \& Lucchinetti, C. F. (2012). Pathology of demyelinating diseases. Annual Review of Pathology: Mechanisms of Disease, 7, 185-217. [DOI:10.1146/annurevpathol-011811-132443] [PMID]

Qi, Z., He, J., Su, Y., He, Q., Liu, J., \& Yu, L., et al. (2011). Physical exercise regulates $\mathrm{p} 53$ activity targeting $\mathrm{SCO} 2$ and increases mitochondrial COX biogenesis in cardiac muscle with age. PloS One, 6(7), e21140. [DOI:10.1371/journal.pone.0021140] [PMID]

Queipo-Ortuño, M. I., Seoane, L. M., Murri, M., Pardo, M., Gomez-Zumaquero, J. M., \& Cardona, F., et al. (2013). Gut microbiota composition in male rat models under different nutritional status and physical activity and its association with serum leptin and ghrelin levels. PloS One, 8(5), e65465. [DOI:10.1371/journal.pone.0065465] [PMID] [PMCID]

Rahmanzadeh, R., Sahraian, M. A., Rahmanzade, R., \& Rodriguez, M. (2018). Demyelination with preferential MAG loss: A complex message from MS paraffin blocks. Journal of The Neurological Sciences, 385, 126-130. [DOI:10.1016/j.jns.2017.12.029] [PMID] 
Ritchie, I. R., MacDonald, T. L., Wright, D. C., \& Dyck, D. J. (2014). Adiponectin is sufficient, but not required, for exercise-induced increases in the expression of skeletal muscle mitochondrial enzymes. The Journal of Physiology, 592(12), 26532665. [DOI:10.1113/jphysiol.2014.273680] [PMID] [PMCID]

Robinson, A. P., Harp, C. T., Noronha, A., \& Miller, S. D. (2014). The experimental autoimmune encephalomyelitis (EAE) model of MS: Utility for understanding disease pathophysiology and treatment. Handbook of Clinical Neurology, 122, 173-189. [DOI:10.1016/B978-0-444-52001-2.00008-X] [PMID] [PMCID]

Ruan, Y. (2001). A population of nestin-immunoreactive neuron are distintict from choline acetyltransferase and NADPHdiaphorese neurons in the medial septum-diagonal band complex of adult rat basal forebrain. Anat. Res.(China), 23, 1-4.

Ruckh, J. M., Zhao, J. W., Shadrach, J. L., van Wijngaarden, P., Rao, T. N., \& Wagers, A. J., et al. (2012). Rejuvenation of regeneration in the aging central nervous system. Cell Stem Cell, 10(1), 96-103. [DOI:10.1016/j.stem.2011.11.019] [PMID] [PMCID]

Sakalidou, M., Leibig, N., Boyle, V., Koulaxouzidis, G., \& Penna, V. (2011). Interleukin-10 and regeneration in an end-to-side nerve repair model of the rat. Journal of the Peripheral Nervous System, 16(4), 334-340. [DOI:10.1111/j.1529-8027.2011.00368.x] [PMID]

Su, P. H., Chen, C. C., Chang, Y. F., Wong, Z. R., Chang, K. W., \& Huang, B. M., et al. (2013). Identification and cytoprotective function of a novel nestin isoform, Nes-S, in dorsal root ganglia neurons. The Journal of Biological Chemistry, 288(12), 8391-8404. [DOI:10.1074/jbc.M112.408179] [PMID] [PMCID]

Sugiura, H., Sugiura, H., Nishida, H., Inaba, R., Mirbod, S. M., \& Iwata, H. (2001). Effects of different durations of exercise on macrophage functions in mice. Journal of Applied Physiology, 90(3), 789-794. [DOI:10.1152/jappl.2001.90.3.789] [PMID]

Tomlinson, L., Huang, P. H., \& Colognato, H. (2018). Prefrontal cortex NG2 glia undergo a developmental switch in their responsiveness to exercise. Developmental Neurobiology, 78(7), 687-700. [DOI:10.1002/dneu.22590] [PMID

van Praag, H., Kempermann, G., \& Gage, F. H. (2000). Neural consequences of enviromental enrichment. Nature Reviews Neuroscience, 1(3), 191-198. [DOI:10.1038/35044558] [PMID]

Watanabe, M., Toyama, Y., \& Nishiyama, A. (2002). Differentiation of proliferated NG2-positive glial progenitor cells in a remyelinating lesion. Journal of Neuroscience Research, 69(6), 826-836. [DOI:10.1002/jnr.10338] [PMID]

Zhang, F., Wu, Y., \& Jia, J. (2011). Exercise preconditioning and brain ischemic tolerance. Neuroscience, 177, 170-176. [DOI:10.1016/j.neuroscience.2011.01.018] [PMID]

Zimmerman, L., Parr, B., Lendahl, U., Cunningham, M., McKay, R., \& Gavin, B., et al. (1994). Independent regulatory elements in the nestin gene direct transgene expression to neural stem cells or muscle precursors. Neuron, 12(1), 11-24 [DOI:10.1016/0896-6273(94)90148-1] [PMID] 